\title{
The Effect of Bedside Exercise Program on Stroke Patients with Dysphagia
}

\author{
Jung-Ho Kang, M.D., Rae-Young Park, M.D., Su-Jin Lee, M.D., Ja-Young Kim, M.D., \\ Seo-Ra Yoon, M.D, Kwang-Ik Jung, M.D.
}

Department of Rehabilitation Medicine, Gwangju Veterans Hospital, Gwangju 506-705, Korea

\begin{abstract}
Objective To examine the effects of a bedside exercise program on the recovery of swallowing after a stroke.
Method Fifty stroke patients with dysphagia ( $<6$ months post-stroke) were enrolled and classified into two groups, the experimental ( 25 subjects) and control groups ( 25 subjects). The control group was treated with conventional swallowing therapy. The experimental group received additional bedside exercise training, which consisted of oral, pharyngeal, laryngeal, and respiratory exercises, 1 hour per day for 2 months, and they were instructed regarding this program through the nursing intervention. All patients were assessed for their swallowing function by Videofluoroscopic Swallowing Study (VFSS), using the New VFSS scale, the level of functional oral intake, the frequency of dysphagia complications, the presence (or not) of tube feeding, the mood state and quality of life before the treatment and at 2 months after the treatment.

Results After 2 months of treatment, the experimental group showed a significant improvement in the swallowing function at the oral phase in the New VFSS Scale than that of the control group $(\mathrm{p}<0.05)$. Further, they also showed less depressive mood and better quality of life than the control group. However, there was no significant change in the incidence of dysphagia complication and the presence (or not) of tube feeding between the two groups.

Conclusion Bedside exercise program showed an improvement of swallowing function and exhibited a positive secondary effect, such as mood state and quality of life, on subacute stroke patients with dysphagia. For improvement of rehabilitation results on subacute stroke patients with dysphagia, this study suggests that additional intensive bedside exercise would be necessary.
\end{abstract}

Key Words Dysphagia, Videofluoroscopic swallowing study, Bedside exercise

Received September 26, 2011; Accepted June 8, 2012

Corresponding author: Seo-Ra Yoon

Department of Rehabilitation Medicine, Gwangju Veterans Hospital, Sanwol-dong, Gwangsan-gu, Gwangju 506-705, Korea

Tel: +82-62-602-6295, Fax: +82-62-602-6990,E-mail: standupmd@ hanmail.net

(C) This is an open-access article distributed under the terms of the Creative Commons Attribution Non-Commercial License (http:// creativecommons.org/licenses/by-nc/3.0) which permits unrestricted noncommercial use, distribution, and reproduction in any medium, provided the original work is properly cited.

Copyright $\odot 2012$ by Korean Academy of Rehabilitation Medicine

\section{INTRODUCTION}

Dysphagia is one of the most common complications, which may occur after a stroke, and it occurs to approximately $37-78 \%$ of patients with a stroke. ${ }^{1,2}$ This may lead to a degraded quality of life, as well as cause malnutrition, dehydration, and severe aspiration pneumonia. Severe aspiration pneumonia is life-threatening, and possibly lead to death. ${ }^{3-5}$ Several reports showed that 
stroke-induced dysphagia was naturally resolved, ${ }^{6,7}$ but according to Howard et al., ${ }^{8} 30 \%$ of stoke patients required sustained parenteral nutrition or percutaneous endoscopic gastrostomy, which decreased the overall quality of life. This may result in reduced efficacy of rehabilitation and prolonged hospitalization with negative economic and emotional impacts, and thus, there is a critical need for treatment. ${ }^{9-11}$

For the treatment of dysphagia, various methods and exercise have been introduced and used in clinical practice. The ways to achieve treatment are divided into direct and indirect methods; direct method is associated with the use of foods and achieves treatment through diet adjustments, and diverse posture changes, while indirect method is associated with the stimulation or behavioral swallowing techniques without the direct use of food, which is usually performed when at least $10 \%$ of foods that enter in the mouth are aspirated or takes 10 minutes or longer for the passage through the mouth and pharynx.

One of the indirect methods, stimulation treatment, may increase the swallowing speed and sensitivity by stimulating the inner surface of the oral cavity with laryngoscope or inducing thermal stimulation, ${ }^{12,13}$ and an electrical stimulation, which is the most common method used recently, may result in the change of neuroplasticity through inducing somatic sense or repeat movement of the laryngeal elevator muscle. ${ }^{14-17}$ In addition to the stimulation treatment, exercise can be combined, which consisted of oral, pharyngeal, laryngeal and respiratory exercises. For oral exercise, exercises with lips, tongue and jaw can be conducted separately, while pharyngeal exercise can be done through tongue pulling, strengthening of pharyngeal wall, soft palate exercise, Masako maneuver and Shaker exercise. Laryngeal exercise is conducted to improve the airway obstruction and voice clarification through exercises, including airway closure, vocal cords adduction, false vocal cord enhancement, and respiration exercise for swallowing facilitation, which includes glottis swallow, Mendelsohn and effortful swallow.

Recently, a number of studies have been conducted to demonstrate the efficacy of current treatment methods for dysphagia. Freed et al. ${ }^{16}$ has reported that an electrical stimulation is effective for dysphagia treatment, and Rosenbek et al. ${ }^{12}$ and Hamdy et al. ${ }^{13}$ have demonstrated the effectiveness of thermal and chemical stimulation.
In addition, the effects of effortful swallow, ${ }^{18}$ and the Mendelsohn maneuver were demonstrated, and Shaker reported that the shaker exercise named after own name was effective for the treatment of dysphagia. ${ }^{19-21}$ Like these, majority of the studies regarding dysphagia treatment was limited to an electrical or thermal stimulation, or tended to demonstrate its effect focusing on the limited number of exercises.

This exercise program for dysphagia was designed, with the need of comprehensive exercise programs, rather than maintaining one exercise, in combination with the stimulation treatment for the actual treatment for patients with swallowing disorders, as well as with the needs of an intensive program, which can be conducted in a ward, considering insufficient time spent in occupational therapy and reduced compliance of patients and caregivers. Further, we intended to determine the direct effects of the exercise program on the swallowing capacity and indirect effects on depressive symptoms and quality of life, which may affect the recovery outcome of poststroke function.

\section{MATERIALS AND METHODS}

\section{Subjects}

This study was conducted in 25 patients with stroke who had been hospitalized at the rehabilitation department of our hospital, between 2009 and 2010. The inclusion criteria were patients who had an onset of stroke within 6 months; whose dysphagia was confirmed by VFSS; who was capable of communication and fairly good understanding; and who can follow instructions, which consisted with at least one step. The exclusion criteria were any patients with a previous history of other diseases, which may have caused dysphagia; who had severe cognitive disorder, such as dementia; who cannot carry out video fluoroscopy due to incapability of sitting posture; or who was not able to follow study instructions. Moreover, 25 patients were allocated to the control group, considering sex, age, swallowing capability, daily activity performance and stroke location.

\section{Method}

For patients in both experimental and control groups, physical examination and VFSS were carried out to evaluate the baseline dysphagia level at admission. Physical 
examination was conducted with an assessment using dysphagia bedside scale, which consists of lips pursing, chewing, tongue motility, facial sense, laryngeal elevation and reflex coughing. To determine the improvement of swallowing function by making a comparison of before and after the treatment, and the video fluoroscopic swallowing study was performed prior to and at 2 months after the treatment for dysphagia. Modified VFSS based on Logeman ${ }^{22}$ protocol was conducted and recorded, and then interpreted by an agreement of two rehabilitation specialists. Although it was a qualitative test, based on the findings of interpreting doctors, for quantitative determination of the treatment effects, a new VFSS scale developed by Jung et al. ${ }^{23}$ was employed. After $140 \mathrm{~g} / 100$ $\mathrm{ml}$ of Solotop sol 140 (Taejoon Pharma Co., Seoul, Korea) was diluted with 3 -fold volume of water to $35 \% / v$, results with $2 \mathrm{ml}$ of the solution were analyzed for each parameter in normal speed and frame unit, by two rehabilitation specialists. Compared to the previous functional dysphagia scale, the new VFSS scale enables a detailed analysis on the findings in the oral stage, such as lips closure, food residues after swallowing in the mouth, oral passage time, trough formation, as well as the consideration of the findings in the pharyngeal stage, including laryngeal elevation, laryngeal closure, presence of invasiveness, food residues after swallowing in the pyriform sinus and epiglottic vallecular sinus, and passage time to the larynx.

Both the experimental and control groups visited the occupational therapy room for $\mathbf{3 0}$ minutes a day, 5 days a week for two months to carry out a tactile-thermal stimulation. For the experimental group, additional exercise program for dysphagia treatment was conducted under nursing intervention for at least 1 hour a day, by an average during the 2-month study period. The exercise program was composed with oral, pharyngeal, laryngeal and respiration exercises. The needs of an additional exercise to improve the inadequate stage of swallowing, which were confirmed by a video fluoroscopy, were explained to and recognized by the patients and their caregivers to promote further efforts for this exercise. The oral exercise included lips, tongue and jaw exercises, and the oralpharyngeal exercise included tongue movement, including puling and reaching soft palate with the tip and soft palate exercise, such as yawning and straw blowing, as well as the Shaker exercise. With the expectation of preventing aspiration pneumonia, laryngeal exercises were performed, including airway closure, vocal cord adduction and breathing exercises. At last, for the respiration exercise to facilitate swallowing, effortful swallowing and supraglottis swallowing were exercised. For an effective implementation of the bedside exercise, rehabilitation specialists and occupational therapists provided training for nurses. Video recording of the swallowing exercise were played in the wards for the patients and their caregivers, and the daily implementation was checked by the nurses.

To determine the effects of the bedside exercise, video fluoroscopy was conducted, using the new VFSS scale before and after the exercise to compare the swallowing functions in the oral and pharyngeal stages, as well as the Functional oral intake scale (FOIS), transition from the tube feeding to oral feeding, and incidence of aspiration pneumonia were compared between the two groups.

Table 1. Demographic of Subjects

\begin{tabular}{|c|c|c|c|}
\hline Variable & Experimental & Control & $\begin{array}{c}\text { p- } \\
\text { value }\end{array}$ \\
\hline $\operatorname{Sex}(M: F)$ & $16: 09$ & $18: 07$ & 0.54 \\
\hline Age & $68.3 \pm 6.6$ & $66.7 \pm 6.01$ & 0.35 \\
\hline Cause of stroke (No.) & & & 0.56 \\
\hline Hemorrhage & 10 & 8 & \\
\hline Infarction & 15 & 17 & \\
\hline Brain lesion (No.) & & & 0.54 \\
\hline Brain stem & 9 & 7 & \\
\hline Non-Brain stem & 16 & 18 & \\
\hline $\begin{array}{l}\text { Clinical dysphagia } \\
\text { scale }\end{array}$ & $52.3 \pm 7.5$ & $50.1 \pm 6.8$ & 0.42 \\
\hline FIM & $67.7 \pm 10.9$ & $68.4 \pm 9.3$ & 0.82 \\
\hline MMSE & $16.0 \pm 3.1$ & $17.1 \pm 3.2$ & 0.25 \\
\hline \multicolumn{4}{|l|}{ Tube feeding } \\
\hline No. & 12 & 11 & 0.78 \\
\hline T-FIM & $61.2 \pm 8.6$ & $63.7 \pm 6.8$ & 0.46 \\
\hline $\begin{array}{l}\text { T-Clinical dyspha- } \\
\text { gia scale }\end{array}$ & $56.2 \pm 7.7$ & $56.6 \pm 4.1$ & 0.85 \\
\hline
\end{tabular}

Baseline demographics and clinical characteristics were compared using the Mann-Whitney U test for numeric data and chi square test (sex, cause of stroke, brain lesion) for nominal data

Values are mean \pm standard deviation

FIM: Functional independent measurement, MMSE: Minimal mental state exam, T-FIM: FIM of tube feeding group, T-Clinical dysphagia scale: Clinical dysphagia scale of tube feeding group 
Moreover, inpatient depressive symptoms, before and after the treatment, were examined using a Beck depression inventory (BDI) and quality of life was assessed with the Stroke Specific Quality of Life Scale (SS-QOL).

\section{Statistical analysis}

A commercial statistic program, SPSS 12.0 for windows, was employed; comparison of clinical characteristics used a student's t-test and a comparison of New VFSS scale before and after the treatment used a MannWhitney test and depressive symptom index. Further, the change in the quality of life was tested with a Wilcoxon signed rank test. The statistical significance level was $\mathrm{p}<0.05$.

\section{RESULTS}

\section{General characteristics of subjects}

The mean ages of the experimental and control groups were 68.3 and 66.7 , respectively. There was no significant difference in the baseline swallowing capacity, daily activity performance and stroke location (Table 1).

\section{Improvement of swallowing function}

After the 2-month treatment for dysphagia, both groups showed an improvement in the swallowing capacity, and the experimental group, with combined exercise program, showed a statistically significant improvement, compared to the control group. In particular, oral stage, assessing mastication, bolus formation, apraxia, and oral transit time (OTT) had meaningful outcomes, compared to that of the control group, while the pharyngeal phase did not have any significant difference between the two groups (Table 2). Also, there was no significant difference between the groups in the incidence of aspiration pneumonia, which is a common complication of dysphagia, and transmission from tube feeding to oral feeding, but in FOIS (Functional oral intake scale), the experimental group showed significant improvement, compared to that of the control group (Table 3).

Table 2. Treatment Outcomes of the New VFSS Scale

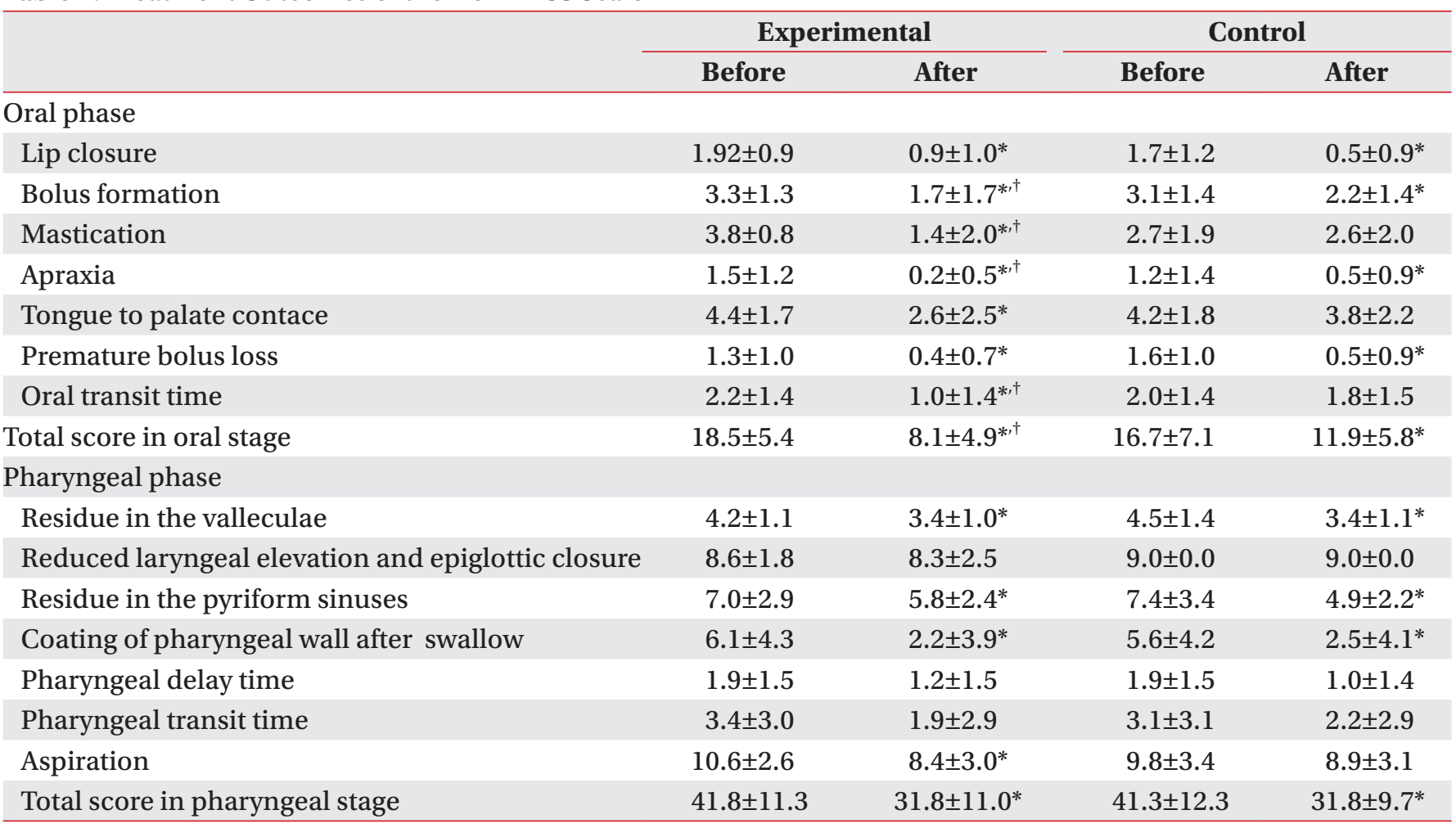

Values are mean \pm standard deviation

VFSS : Videofluoroscopic swallowing study

${ }^{*} \mathrm{p}<0.05$ by Wilcoxon signed rank test: compared to before in each group. ${ }^{\dagger} \mathrm{p}<0.05$ by Mann-Whitney test: compared to Control 
Intensity of depressive symptoms and quality of life

The exercise program was conducted in a ward, simultaneously for patients complaining of swallowing disorder, which resulted in significant decrease of BDI score in the experimental group, from 31.5 to 26.8 through the treatment, while the control group did not show any significant difference between the baseline and Month 2 scores. Also, the assessment with SS-QOL showed significant improvement in relation with parameters of energy, family role and mood in the experimental group, compared to the control group. (Table 4$)(\mathrm{p}<0.05)$.

\section{DISCUSSION}

Swallowing is a complicate mechanism, requiring a cooperation of multiple muscles in the mouth, pharynx, larynx, and esophagus, through the complex neural circuit. Sensory information concerning the foods in the mouth is transferred by the trigeminal, glossopharyngeal, vagus, and facial hypoglossal nerves to the swallowing center, which is located within the nucleus of the solitary tract in the brainstem and cerebral hemisphere. When the swallow response is initiated with muscular contraction in the mouth, pharynx and larynx, it causes messages to be sent to the trigeminal, facial, glosso-

Table 3. Outcomes of Bedside Dysphagia Exercise

\begin{tabular}{lcccccc} 
& \multicolumn{2}{c}{ Experimental } & & \multicolumn{2}{c}{ Control } \\
\cline { 2 - 4 } \cline { 5 - 7 } & Before & After & & Before & After \\
\hline FOIS & $2.8 \pm 1.4$ & $4.6 \pm 1.0^{*, \dagger}$ & & $2.6 \pm 1.5$ & $3.6 \pm 1.2^{*}$ \\
Aspiration Pneumonia & & 5 & & & 6 & 7 \\
Tube present (No.) & 12 & 6 & & 11 & $72.9 \pm 9.9^{*}$ \\
FIM score & $68.4 \pm 9.3$ & $74.2 \pm 7.5^{*}$ & & $67.7 \pm 10.9$ & $29.2 \pm 4.2$ \\
\hline BDI & $31.5 \pm 5.8$ & $26.8 \pm 6.0^{*}$ & & $30.0 \pm 5.7$ & \\
\hline
\end{tabular}

Values are mean \pm standard deviation

FOIS: Functional oral intake scale, FIM: Functional independent measurement, BDI: Beck depression inventory ${ }^{*} \mathrm{p}<0.05$ by Wilcoxon signed rank test: compared to before in each group. ${ }^{\dagger} \mathrm{p}<0.05$ by Mann-Whitney test : compared to Control

Table 4. Stroke-Specific Quality of Life

\begin{tabular}{|c|c|c|c|c|}
\hline & \multicolumn{2}{|c|}{ Experimental } & \multicolumn{2}{|c|}{ Control } \\
\hline & Before & After & Before & After \\
\hline Energy & $5.68 \pm 2.1$ & $9.8 \pm 1.7^{*, \dagger}$ & $6.7 \pm 2.4$ & $7.2 \pm 2.0$ \\
\hline Family role & $5.9 \pm 2.3$ & $8.1 \pm 1.9 *$ & $6.9 \pm 2.3$ & $7.4 \pm 2.4$ \\
\hline Language & $15.7 \pm 5.1$ & $16.4 \pm 4.7$ & $16.0 \pm 3.7$ & $17.6 \pm 3.3^{*}$ \\
\hline Mobility & $15.2 \pm 6.0$ & $17.4 \pm 5.0^{*}$ & $15.1 \pm 4.9$ & $16.4 \pm 5.0^{*}$ \\
\hline Mood & $11.5 \pm 2.9$ & $15.1 \pm 2.3^{*, \dagger}$ & $12.1 \pm 3.5$ & $12.6 \pm 3.3$ \\
\hline Personality & $7.1 \pm 2.3$ & $8.0 \pm 2.4$ & $7.6 \pm 1.8$ & $8.2 \pm 2.1$ \\
\hline Self care & $11.8 \pm 4.5$ & $13.7 \pm 4.0^{*}$ & $12.4 \pm 3.4$ & $13.9 \pm 3.5^{*}$ \\
\hline Social roles & $11.2 \pm 3.4$ & $12.2 \pm 2.5$ & $11.8 \pm 3.1$ & $13 \pm 3.4^{*}$ \\
\hline Thinking & $6.8 \pm 2.0$ & $8.7 \pm 2.2^{*}$ & $7.9 \pm 1.8$ & $9.2 \pm 2.3^{*}$ \\
\hline Upper extremity function & $14.0 \pm 4.3$ & $15.3 \pm 4.3^{*}$ & $13.2 \pm 2.4$ & $14.9 \pm 2.5^{*}$ \\
\hline Vision & $13.1 \pm 2.5$ & $13.6 \pm 2.2$ & $12.8 \pm 1.2$ & $13.6 \pm 1.3$ \\
\hline Work & $7.6 \pm 2.7$ & $9.3 \pm 2.6^{*}$ & $7.4 \pm 2.0$ & $9.4 \pm 1.6^{*}$ \\
\hline Total score & $125.5 \pm 31.4$ & $147.7 \pm 22.9^{*}$ & $129.8 \pm 20.1$ & $144.5 \pm 24.7^{*}$ \\
\hline
\end{tabular}

Values are mean \pm standard deviation

${ }^{*} \mathrm{p}<0.05$ by Wilcoxon signed rank test: compared to before in each group. ${ }^{\dagger} \mathrm{p}<0.05$ by Mann-Whitney test: compared to Control 
pharyngeal, vagus and hypoglossal nerves. ${ }^{24-26}$ If the swallowing-related nerves, listed above, are injured after the incidence of stroke, swallowing disorder is caused. Recovery of the swallowing disorder can be achieved by the recovery of an injured nerve, as well as the recovery of the strength and cooperation of muscles involved in the swallowing process. Therefore, the program of repeat exercise for dysphasia treatment can induce the change in neuroplasticity, and contribute to an increased volume and strength of muscles and enhanced cooperation of the affected swallowing muscles, which will improve the swallowing capacity. ${ }^{27}$

Normal swallowing processes are divided into oral, pharyngeal and esophageal stages. When stroke causes an injury in a stage, the pattern of the swallowing disorder depends on the affected stage. Stoke in the brainstem is associated with high possibility of injury in the swallowing center, and thus, may cause severe dysphagia with risk of food aspiration to the airway and lots of remained foods in the pharynx after the end of the swallowing reflex. Stoke in the cerebral hemisphere causes greater problems in the oral stage, compared to one that occurs in the brainstem, and this is also associated with problems of swallowing reflex in pharyngeal stage. ${ }^{28}$ The exercise program for dysphagia treatment includes oral, pharyngeal, laryngeal and respiration exercises, and appropriate exercise can be selected in consideration of the swallowing stage. Further, it ensures the more intensive exercise targeting the weaker stage by using a video fluoroscopy.

To verify the effectiveness of the exercise program, this study compared the swallowing function between the two groups, prior to and at 2 months after the treatment, using the new video fluoroscopy study scale (new VFSS scale), recommended by Jung et al. ${ }^{23}$ Separate assessment on the oral stage and pharyngeal stage found that statistically significant improvement of the new VFSS scale was shown only in the oral stage, compared to that of the control group, and the improvement was noticeable particularly in the Bolus formation, mastication, apraxia and OTT (Table 2). The oral stage starts when the food comes into the mouth and lasts until the food bolus is moved to the pharynx by the tongue and the swallowing reflex is initiated, which requires a normal tension and mobility of the tongue. Because the oral stage is voluntary, unlike the pharyngeal and esophageal stages, the repeat exer- cises of the lips, tongue and jaw in the program would help the recovery of muscular tension and mobility, and thus, it was believed that the transition process from the oral stage to the pharyngeal stage was improved.

On the contrary, pharyngeal stage did not show any significant difference in the new VFSS scale, compared to the control group. The pharyngeal phase, where the swallowing reflex occurred, is processed via a series of activities to prevent the aspiration into the airway, such as folding epiglottis, closure of vocal cord and closure of the laryngeal vestibule and other activities, including pharyngeal peristalisis and relaxation of cricopharyngeous muscle. This stage is mainly controlled by the swallowing center, which shows autonomic activities and it is also known to have voluntary factors. ${ }^{24}$ Electrical stimulation, conducted in both groups, provided somatic sense and induced repeat movement of the laryngeal elevator muscle, which contributed to the improvement in the pharyngeal stage in both groups, and the experimental group with additional exercise did not show any significant difference, compared to that of the control group.

In the previous studies, Shaker et al. ${ }^{21}$ has reported that isometric and isotonic head lift activities to strengthen the submental musculature induced improvements in the upper esophageal sphincter opening, and Ding et al. ${ }^{19}$ has reported that Mendelsohn maneuver was helpful in the laryngeal elevation and cricopharyngeal opening. Furthermore, Robbins et al. ${ }^{27}$ used a progressive lingual strength program and demonstrated improvements in the swallowing pressures, airway protection, and lingual volume. However, most previous studies incorporate some exercises principles, and no one considers the multiple exercise principles and other dysphagia strategies, such as electrical stimulation and so on. We tried to apply a comprehensive bedside exercise-based therapy framework with using the conventional dysphagia strategies for the treatment of dysphagia in stroke patients.

In this study, the comprehensive bedside dysphagia exercises did not show any significant difference in the pharyngeal stage, compared to that of the control group. As a possible reason that explains the results, the current electrical stimulation treatment and pharyngeal deep nerve stimulation can be considered more effective than the exercise treatment. However, we thought that because the pharyngeal stage is involuntary, controlled by the swallowing center in the brainstem, exercise of 
the voluntary movement is difficult, and thus, the subsequent improvement of muscular strength and change in neuroplasticity may slow down.

In the comprehensive exercise program for dysphagia treatment, the overall score of the new VFSS scale has been significantly improved, compared to that of the controlled group. However, there was no meaningful difference in the transmission to oral feeding or incidence of aspiration pneumonia. We expected a decrease in pneumonia incidence with eliminated foods in the airway and enhanced swallowing capacity achieved by laryngeal exercise, but patients with relatively delayed recovery of function showed poor outcomes, due to aspiration pneumonia, which caused reduced compliance with the exercise program, and thus, there were no meaningful effects from the program.

Gillen et al. ${ }^{29,30}$ and Morris et al. ${ }^{31}$ have reported that patients with post-stroke depression showed delayed functional recovery, compared to those without depression, and this was because of the depression-induced lower motivation and various cognitive symptoms associated with learning and processing of information. In addition, You and $\mathrm{Ann}^{32}$ reported that motivation for rehabilitation and quality of life were correlated, and thus, depressive symptoms and quality of life can be considered as major factors that may affect the effectiveness of rehabilitation. When comparing the patient groups, with tube feeding and oral feeding, a group with tube feeding showed higher depression and lower quality of life (Fig. 1). Although the other factors, which may affect the level of depression and quality of life, had not been considered, the way of feeding can be a critical factor that may affect patient's depression and quality of life. The exercise program did not show any significant difference in the removal of tube for nutrition, but the group participating in the exercise program showed a meaningful improvement in the depressive symptoms and quality of life, in particular, the energy, family role and mood.

The results of swallowing capacity assessment, using VFSS, showed a significant improvement in the bolus formation, mastication, apraxia and OTT for the oral phase, which contributed to the favored results in FOIS, compared to the control group. Unlike the results of VFSS, FOIS includes subjective items that patients and caregivers can directly feel, and thus, improvement of FOIS would have a positive influence on the motivation for the participation in the exercise program and the quality of life. Moreover, during the exercise program, patient's condition was periodically acknowledged to the patient and the caregiver, and the program was conducted as a group therapy for patients, caregivers and other patients in the same ward, which would contribute to the motivation of the patients for rehabilitation, and improved quality of life and depressive symptoms.

The study had several limitations. Due to a small population size, location of the brain lesion, such as brain stem, which may affect the pattern of dysphagia and the degree of recovery, cannot be considered. As pa-
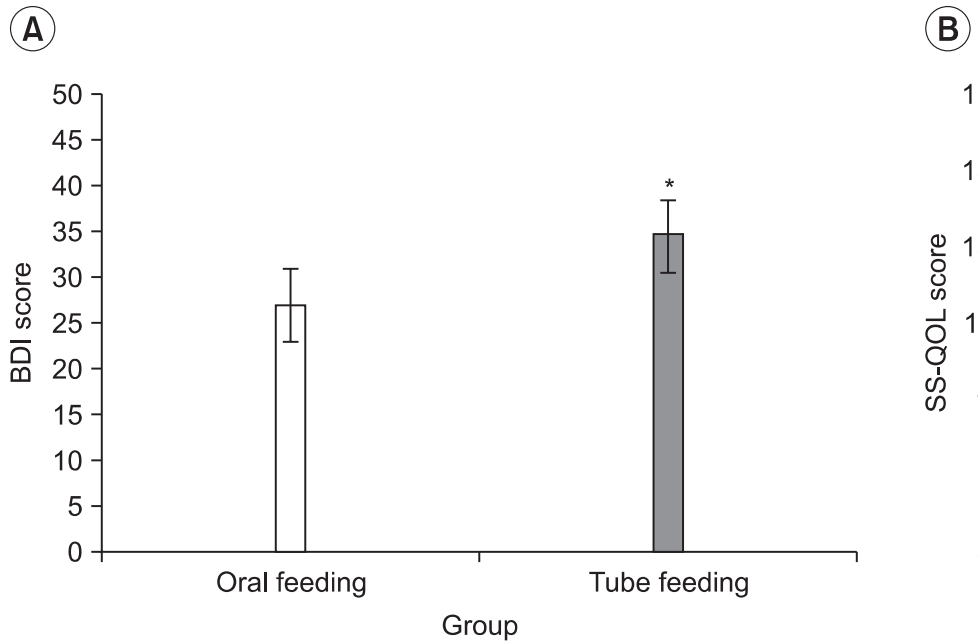

Fig. 1. Depressive mood state and quality of life according to feeding method (oral or tube feeding). (A) Beck Depressive Inventory (BDI) (B) Stroke Specific-Quality of Life (SS-QOL). In the oral feeding group, BDI score was significantly lower and SS-QOL score was higher than those of tube feeding group. * $\mathrm{p}<0.05$ by Mann-Whitney U test: compared to Control. 
tients who were capable of participating in the exercise program were selected, patients with severe dysphagia were excluded, and the patients with tracheostomy tube, which can affect the swallowing function, were also excluded. Moreover, an indirect training method, which was employed dominantly, interfered with the effective dynamic combination with direct and indirect methods, depending on the degree of dysphagia.

\section{CONCLUSION}

We aimed to determine the effectiveness of bedside exercise program for dysphagia treatment. The swallowing capacity was assessed using a new VFSS scale and the experimental group showed a statistically significant difference in the oral stage, compared to that of the control group and their depression symptoms, along with the quality of life were improved from the baseline. Although the small number of population interfered with the generalization of the study result, the study was was meaningful in the aspect that comprehensively intensive bedside exercise were designed and conducted via an additional nursing intervention, and this exercise program could be a useful modality to rehabilitate the dysphagia patients, especially at the oral phase during swallowing. In addition, this study could be the foundation of largescaled studies to improve the effective exercise program for dysphagia and to develop the implementation plan in the future.

\section{REFERENCES}

1. Katzan IL, Cebul RD, Husak SH, Dawson NV, Baker DW. The effect of pneumonia on mortality among patients hospitalized for acute stroke. Neurology 2003; 60: 620-625

2. Alberts MJ, Horner J, Gray L, Brazer SR. Aspiration after stroke: lesion analysis by brain MRI. Dysphagia 1992; 7: 170-173

3. Chokshi SK, Asper RF, Khandheria BK. Aspiration pneumonia: a review. Am Fam Physician 1986; 33: 195-202

4. Grober ME. Dysphagia: diagnosis and management, 3rd ed, Newton: Butterworth-Heinemann, 1997, 139143

5. Kikuchi R, Watabe N, Konno T, Mishima N, Sekizawa
K, Sasaki H. High incidence of silent aspiration in elderly patients with community-acquired pneumonia. Am J Respir Crit Care Med 1994; 150: 251-253

6. Han TR, Paik NJ, Park JW, Kwon BS. The prediction of persistent dysphagia beyond six months after stroke. Dysphagia 2008; 23: 59-64

7. Smithard DG, O'Neill PA, England RE, Park CL, Wyatt R, Martin DF, Morris J. The natural history of dysphagia following a stroke. Dysphagia 1977; 12: 188-193

8. Howard L, Ament M, Fleming CR, Shike M, Steiger E. Cerrent use and clinical outcome of home parenteral and enteral nutrition therapies in the United States. Gastroenterology 1995; 109: 355-365

9. Holas MA, DePippo KL, Reding MJ. Aspiration and relative risk of medical complications following stroke. Arch Neurol 1994; 51: 1051-1053

10. Horner J, Buoyer FG, Alberts MJ, Helms MJ. Dysphagia following brain-stem stroke. Clinical correlates and outcome. Arch Neurol 1991; 48: 1170-1173

11. Horner J, Massey EW. Silent aspiration following stroke. Neurology 1988; 38: 317-319

12. Rosenbek JC, Robbins J, Fishback B, Levine RL. Effects of thermal application on dysphagia after stroke. J Speech Hear Res 1991; 34: 1257-1268

13. Hamdy S, Jilani S, Price V, Parker C, Hall N, Power M. Mudulation of human swallowing behaviour by thermal and chemical stimulation in health and after brain injury. Neurogastroenterol Motil 2003; 15: 69-77

14. Oh BM, Kim DY, Paik NJ. Recovery of swallowing function is accompanied by the expansion of the cortical map. Int J Neurosci 2007; 117: 1215-1227

15. Ludlow CL, Humbert I, Saxon K, Poletto C, Sonies B, Crujido L. Effects of surface electrical stimulation both at rest and during swallowing in chronic pharyngeal dysphagia. Dysphagia 2007; 22: 1-10

16. Freed ML, Freed L, Chatburn RL, Christian M. Electrical stimulation for swallowing disorders caused by stroke. Respir Care 2001; 46: 466-474

17. Park CL, O'Neil PA, Martin DF. A pilot exploratory study of oral electrical stimulation on swallow function following stroke: an innovative technique. Dysphagia 1997; 12: 161-166

18. Bulow M, Olsson R, Ekberg O. Videomanometric analysis of supraglottic swallow, effortful swallow, and chin tuck in patients with pharyngeal dysfunction. Dysphagia 2001; 16: 190-195 
19. Ding R, Larson CR, Logemann JA, Rademaker AW. Surface electromyographic and electroglottographic studies in normal subjects under two swallow conditions: normal and during the Mendelsohn maneuver. Dysphagia 2002; 17: 1-12

20. Shaker R, Kern M, Bardan E, Taylor A, Stewart ET, Hoffmann RG, Arndorfer RC, Hofmann C, Bonnevier J. Augmentation of deglutitive upper esophageal sphincter opening in the elderly by exercise. Am J Physiol 1997; 272: 1518-1522

21. Shaker R, Easterling C, Kern M, Nitschke T, Massey B, Daniels S, Grande B, Kazandjian M, Dikeman K. Rehabilitation of swallowing by exercise in tube-fed patients with pharyngeal dysphagia secondary to abnormal UES opening. Gastroenterology 2002; 122: 1314-1321

22. Logeman JA. Evaluation and treatment of swallowing disorders, 2nd ed, San Diego: College Hill Press, 1983, 168-180

23. Jung SH, Lee KJ, Hong JB, Han TR. Validation of clinical dysphagia scale: based on videofluoroscopic swallowing study. J Korean Acad Rehab Med 2005; 29: 343350

24. Ertekin C, Aydogdu I. Neurophysiology of swallowing. Clin Neurophysiol 2003; 114: 2226-2244

25. Jean A. Brain stem control of swallowing: neuronal network and cellular mechanisms. Physiol Rev 2001;
81: 929-969

26. Lund JP, Kolta A, Westberg KG, Scott G. Brainstem mechanisms underlying feeding begaviors. Curr Opin Neurobiol 1998; 8: 718-724

27. Robbins JA, Kays SA, Gangnon RE, Hind JA, Hewitt AL, Gentry LR, Taylor AJ. The effects of lingual exercise in stroke patients with dysphagia. Arch Phys Med Rehabil 2007; 88: 150-158

28. Lee CK, Kim JA. Pattern of post-stroke swallowing disorder according to the brain lesion. J Korean Acad Rehab Med 2001; 25: 193-201

29. Gillen R, Eberhardt TL, Tennen H, Affleck G, Groszmann Y. Screening for depression in stroke: relationship to rehabilitation efficiency. J Stroke Cerebrovasc Dis 1999; 8: 300-306

30. Gillen R, Tennen H, Mckee TE, Gernert-Dott P, Affleck G. Depressive symptoms and history of depression predict rehabilitation efficiency in stroke patiens. Arch phys Med Rehabil 2001; 82: 1645-1649

31. Morris PL, Robinson RG, Andrzejewski P, Samuels J, Price TR. Association of depression with 10-year potstroke mortality. Am J Psychiatry 1993; 150: 124-129

32. You YY, Ann CS. A study of the relationships between perceived rehabilitation-motivation and quality of life in patients after a cerebrovascular accident. KSOT 2009; 17: 1-15 